\title{
Hybrid Iterative Detection and Decoding of Near-Instantaneously Adaptive Turbo-Coded Sparse Code Multiple Access
}

\author{
Yusha Liu, Luping Xiang, Member, IEEE, Robert G. Maunder, Senior Member, IEEE, Lie-Liang Yang, Fellow, \\ IEEE and Lajos Hanzo, Fellow, IEEE
}

\begin{abstract}
Reduced-complexity hybrid detection and decoding (HDD) schemes are conceived for turbo-coded sparse code multiple access (SCMA) by analysing its convergence behavior using extrinsic information transfer (EXIT) charts, which outperforms the state of the art joint detector and decoder (JDD). As a benefit of its carefully controlled complexity, the resultant system is eminently suitable for ultra-reliable low-latency communication (URLLC) in multiuser scenarios, since it requires a low number of turbo iterations between the detector and the decoder In particular, a pair of HDD schemes are proposed. HDD-I and HDD-II achieve a complexity reduction of up to $25 \%$ and $36 \%$, respectively, at a similar bit error rate (BER) performance as that of JDD. Additionally, we propose an adaptive turbo-coded SCMA system for mitigating the influence of multipath propagation so that the system's bits per symbol (BPS) throughput may be improved under favorable channel conditions by using the most appropriate near-instantaneous user load, modulation order and coding rate. Our adaptive system design principle can also be readily used for other channel coding schemes.
\end{abstract}

Index Terms-turbo codes, SCMA, adaptive QAM

\section{INTRODUCTION}

Sparse code multiple access (SCMA) constitutes a typical code-domain non-orthogonal multiple access (NOMA) scheme $[1,2]$, which has been proposed for massive Machine-Type Communication (mMTC) scenarios [3]. By adopting nonorthogonal resource allocation methods, every resource unit is capable of supporting more than one mobile device [46]. In this way, a 'massive' number of connections can be supported by a limited number of resources. The iterative message passing algorithm (MPA) based detection further reduces the complexity of the SCMA system $[7,8]$, compared to optimal maximum likelihood detection.

The SCMA arrangement amalgamated with different channel coding schemes was investigated in [9-15], as summarised in Table I. To be more specific, an iterative multiuser receiver inspired by the 'turbo' principle for turbo-coded SCMA systems was proposed in $[9,15]$ for increasing the coding gain,

The authors are with Electronics and Computer Science, University of Southampton, SO17 1BJ, United Kingdom, e-mail: \{yl6g15,lx1g15,rm,lly,lh\}@soton.ac.uk. (Corresponding author: Luping Xiang)

The financial support of the Engineering and Physical Sciences Research Council project EP/P034284/1 is gratefully acknowledged

L. Hanzo would like to gratefully acknowledge the financial support of the Engineering and Physical Sciences Research Council projects EP/Noo4558/1, EP/P034284/1, COALESCE, of the Royal Society's Global Challenges Research Fund Grant as well as of the European Research Council's Advanced Fellow Grant QuantCom (Grant No. 789028) especially in the case of heavy user loads. Joint detection and decoding (JDD) of a low density parity check (LDPC) coded SCMA system was first proposed by Xiao et al. [10], which achieves a beneficial performance gain over a separate SCMA detector and LDPC decoder. As a further development, a joint factor graph was designed by Han et al. [11] for improving the extrinsic information exchange between the SCMA detector and the LDPC decoder. A similar joint factor graph design was also proposed for a polar-coded SCMA system in [12, 13], which improves the BER performance and reduces the detection complexity by relying on an improved polar code construction.

However, most prior research has been focused on improving the BER, whilst there is a paucity of literature on the complexity and latency improvement of coded SCMA systems. For instance, the end-to-end latency of the 5G new radio (NR) ultra-reliable low latency communication (URLLC) mode [16] must remain within $1 \mathrm{~ms}$ at a frame error rate (FER) of $10^{-5}$ [17], which imposes an extremely stringent constraint on the signal processing time at the receiver. Therefore, improvements are required for these JDD algorithms in terms of latency reduction and throughput increase. Naturally, the channel-coding parameters and the resultant BER, throughput, delay and complexity trade-offs play a pivotal role in this context. It was shown by comparing LDPC, polar and turbo codes that turbo codes exhibit the best BER performance at low to moderate coding rates $[17,18]$, which is promising for providing ultra-high reliability in future URLLC applications, such as augmented reality and automated driving $[19,20]$.

In addition to optimizing the JDD algorithm, another potent technique for improving transmission system performance over multipath fading channels is by using adaptive modulation and coding $[21,22]$, which improves the system's overall throughput during favorable channel conditions. To be more specific, in a turbo-coded SCMA system the different factors influencing system performance may be jointly optimised to combat the deleterious effects of fading. In this way, the throughput improvement in terms of bits per symbol (BPS) will directly result in the reduction of latency.

Motivated by satisfying the URLLC requirement, we first improve the activation order of the SCMA detector and turbo decoder iterations by using extrinsic information transfer (EXIT) chart analysis [23]. We then propose a hybrid detection and decoding (HDD) scheme for our turbo-coded SCMA system, which is capable of reducing latency, while maintaining high 
TABLE I

CONTRASTING THE NOVELTY OF THIS CONTRIBUTION TO THE LITERATURE.

\begin{tabular}{l|c|c|c|c|c|c|c|c}
\hline Contributions & HDD & [9] & [10] & [11] & [12] & [13] & [14] & [15] \\
\hline \hline JDD design & $\checkmark$ & $\checkmark$ & $\checkmark$ & $\checkmark$ & $\checkmark$ & $\checkmark$ & $\checkmark$ & $\checkmark$ \\
\hline EXIT chart Analysis & $\checkmark$ & & & & & $\checkmark$ & & $\checkmark$ \\
\hline User overload investigation & $\checkmark$ & & & & & & & \\
\hline Fading channel performance & $\checkmark$ & $\checkmark$ & $\checkmark$ & & & $\checkmark$ & $\checkmark$ & $\checkmark$ \\
\hline Joint factor graph design & & & $\checkmark$ & $\checkmark$ & $\checkmark$ & $\checkmark$ & & \\
\hline Complexity reduction & $\checkmark$ & & & & $\checkmark$ & & & $\checkmark$ \\
\hline Early termination & $\checkmark$ & & & & & & & \\
\hline BER improvement & $\checkmark$ & $\checkmark$ & $\checkmark$ & $\checkmark$ & & $\checkmark$ & & $\checkmark$ \\
\hline Adaptive system design & $\checkmark$ & & & & & & & \\
\hline
\end{tabular}

reliability. Additionally, a near-instantaneously adaptive system is designed for mitigating multipath effects and for further improving the system's throughput. The main contributions of our paper are summarised as follows.

- First, we investigate the impact of the iterative extrinsic logarithmic likelihood ratio (LLR) exchange between the MPA detector and the logarithmic Bahl-Cocke-JelinekRaviv (LBCJR) turbo decoder by comparing the BER of separate detection and decoding (SDD) to that of the JDD scheme in each iteration. The significant BER improvement of JDD shows the advantage of iterative extrinsic information exchange.

- Second, by analysing the convergence behaviour of EXIT charts, we propose an HDD algorithm which maintains the BER performance compared to the conventional JDD, but achieves a beneficial complexity reduction. To be more specific, we optimize the activation order of detection and decoding scheduling for achieving early termination with the aid of EXIT chart analysis. In this way, the detection and decoding latency can be significantly reduced at a similar BER to that of HDD. Additionally, by exploiting the resultant early-termination property, a pair of HDD examples are proposed, namely, HDD-I and HDD-II, which achieve a complexity reduction of up to $25 \%$ and $36 \%$, respectively, at a similar bit error rate (BER) performance as that of JDD.

- Finally, we propose a near-instantaneously adaptive turbo-coded SCMA system, where the transmitter selects the most appropriate transmission mode according to the prevalent near-instantaneous channel conditions. Our adaptive turbo-coded SCMA system configures itself in the most appropriate mode of operation by jointly selecting the user load, coding rate as well as modulation order by maintaining the data rate at the target BER. Our adaptive system design principle can be readily extended to SCMA systems in combination with other channel coding schemes, such as LDPC codes and polar codes, just to name a few.

The rest of this paper is structured as follows. Section II describes the transmitter and receiver schematics of the turbocoded SCMA system, followed by a state of the art review of the detection and decoding processes in Section III. Section IV provides system performance analysis and proposes HDD for our turbo-coded SCMA system based on EXIT chart analysis. Section $\mathrm{V}$ proposes an adaptive turbo-coded system design example along with characterizing the adaptive system performance employing HDD. Finally, our main conclusions and future research are summarized in Section VI.

\section{System ModeL}

In this section, the transmitter and receiver structures of our turbo-coded SCMA system are detailed in Sections II-A and II-B, respectively, along with our main assumptions and notations.

\section{A. Transmitter}

Let us consider the uplink (UL) of a multiuser system supporting $K$ users simultaneously transmitting their information to a base station (BS) over wireless communication channels with the aid of $N$ orthogonal resources in the time domain $(K \geq N)$, as shown in Fig. 1 . To be more specific, in a turbocoded SCMA system, the $A$ information bits $\boldsymbol{u}_{k}$ transmitted by user $k(1 \leq k \leq K)$ are first turbo encoded into $E$ encoded bits $\boldsymbol{b}_{k}$, resulting in a coding rate of $R=A / E$. Here, we assume that each user transmits the same number of information bits. Note that for LTE turbo codes, $A$ varies from 40 to 6144 bits, in multiples of $8,16,32$ or 64 bits. The turbo encoding process is illustrated in Fig. 1, where two identical convolutional encoders, referred to as the upper and lower encoder, respectively, operate in an identical manner and are connected through a quadratic permutation polynomial (QPP) based interleaver [23, 24]. To be more specific, for LTE turbo codes, the $A$ information bits $\boldsymbol{u}_{k}$ of user $k$, are first convolutional encoded by the upper encoder, which generates $A+3$ parity bits $\boldsymbol{b}_{2, k}$, including three parity termination bits, and three termination bits for $A$ systematic bits $\boldsymbol{b}_{1, k}$. The information bit sequence $\boldsymbol{u}_{k}$ is interleaved by the LTE interleaver [25] and input to the lower encoder of Fig. 1. The interleaved information bits can be denoted as $\overline{\boldsymbol{u}}_{k}=\pi\left(\boldsymbol{u}_{k}\right)$. The lower encoder of Fig. 1 generates only $A+3$ parity bits $\boldsymbol{b}_{3, k}$ and three additional termination bits for $A$ interleaved systematic bits $\overline{\boldsymbol{b}}_{1, k}$. Upon concatenating the three bit frames, namely $\boldsymbol{b}_{1, k}, \boldsymbol{b}_{2, k}$, and $\boldsymbol{b}_{3, k}$, we have a $\bar{E}=3 A+12$-length turbo coded sequence $\overline{\boldsymbol{b}}_{k}$, resulting in an overall coding rate of $\frac{A}{3 A+12}$. By puncturing or repeating some of the coded bits [25], a variable coding rate of $R=A / E \in(0,1)$ can be achieved, resulting in an $E$-bit turbo coded sequence $\boldsymbol{b}_{k}$

After the turbo encoder of Fig. 1, the encoded bits $\boldsymbol{b}_{k}$ are interleaved and mapped by the SCMA codebook $\mathcal{S}_{k}$ of user $k$ to $F=E / \log _{2}(M)$ length- $N$ codewords, each of which 


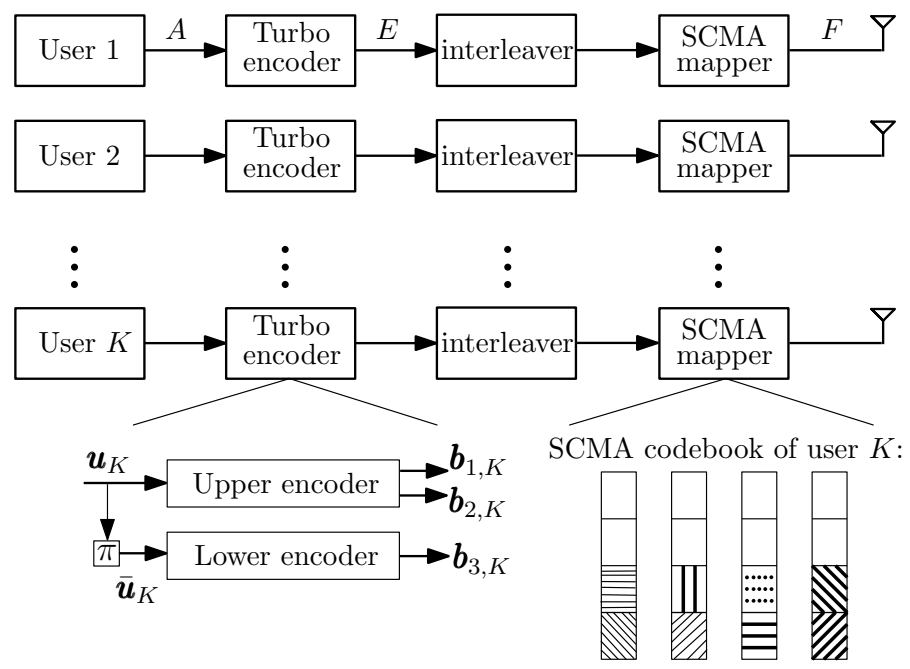

Fig. 1. Transmitter schematic of the turbo-coded SCMA system.

contain only $d_{x}\left(d_{x} \ll N\right)$ non-zero values, where $M$ is the modulation order and the cardinality of $\mathcal{S}_{k}$ is $\left|\mathcal{S}_{k}\right|=M$. The positions of the non-zero elements of the $f$-th SCMA symbol of all $K$ users $\boldsymbol{X}^{(f)}=\left[\boldsymbol{x}_{1}^{(f)}, \boldsymbol{x}_{2}^{(f)} \cdots \boldsymbol{x}_{K}^{(f)}\right]^{T}$ are denoted by the indicator matrix $\bar{V}$ [4]. For example, the most common indicator matrix $\bar{V}$ of an $N \times K=4 \times 6$ SCMA system with $d_{x}=2$ is expressed as

$$
\overline{\boldsymbol{V}}=\left[\begin{array}{llllll}
1 & 1 & 1 & 0 & 0 & 0 \\
1 & 0 & 0 & 1 & 1 & 0 \\
0 & 1 & 0 & 1 & 0 & 1 \\
0 & 0 & 1 & 0 & 1 & 1
\end{array}\right]
$$

The design of the complex values of the non-zero values in (1) is detailed in [26]. Also, we define $d_{c}\left(d_{c} \ll K\right)$ as the number of users that share a single subcarrier, which also indicates the number of non-zero values in a single row of $\overline{\boldsymbol{V}}$.

Let us define the normalised user load for a regular $N \times K$ SCMA system as

$$
\lambda=\frac{K}{N}=\frac{d_{c}}{d_{x}} .
$$

For the SCMA mapper of Fig. 1, the transmitted symbol frame of the $k$-th user becomes:

$$
\boldsymbol{x}_{k}=\left[\boldsymbol{x}_{k}^{(1) T}, \boldsymbol{x}_{k}^{(2) T}, \cdots, \boldsymbol{x}_{k}^{(F) T}\right]^{T} .
$$

\section{B. Receiver}

The receiver schematic of the turbo-coded SCMA system is shown in Fig. 2. We assume that the channel experiences uncorrelated Rayleigh fading, and the channel gains between the $N$ subcarriers of user $k$ and the BS for the $f$-th symbol can be expressed as an $N$-length vector $\boldsymbol{h}_{k}^{(f)}$. Furthermore, we assume that the receiver has perfect knowledge of the channel impulse response. The $N$ received signal observations of the $f$-th symbol in the frame can be expressed as

$$
\boldsymbol{y}^{(f)}=\sum_{k=1}^{K} \operatorname{diag}\left(\boldsymbol{h}_{k}^{(f)}\right) \boldsymbol{x}_{k}^{(f)}+\boldsymbol{n}_{u}
$$

where $\boldsymbol{y}^{(f)}$ is an $N \times 1$ vector and $\boldsymbol{n}_{u}$ is an $N \times 1$ zero-mean complex Gaussian variable vector having a covariance matrix of $2 \sigma^{2} \boldsymbol{I}_{N}$, expressed as $\mathcal{C N}\left(0,2 \sigma^{2} \boldsymbol{I}_{N}\right)$, where $\sigma^{2}=1 /(2 \gamma)$, $\gamma=\left(R \log _{2} M\right) \gamma_{0}$ denotes the signal-to-noise ratio (SNR) per symbol, while $\gamma_{0}$ is the $E_{b} / N_{0}$.

The received signal observations of all $F$ symbols can be expressed as

$$
\boldsymbol{Y}=\left[\boldsymbol{y}^{(1)}, \boldsymbol{y}^{(2)}, \cdots, \boldsymbol{y}^{(F)}\right]
$$

\section{Signal DETECTION AND DECODING}

In this section, we first detail the separate detection and decoding (SDD) algorithm of a turbo-coded SCMA system employing the MPA for SCMA detection and the LBCJR algorithm for turbo decoding. Then JDD is introduced, which exploits the gradually improved LLRs gleaned from the iterative extrinsic information exchange between the MPA detector and LBCJR decoder.

\section{A. Separate signal detection and decoding}

In SDD, MPA detection is first applied to the received signal $Y$, followed by the LBCJR turbo decoder, where the output LLRs of the MPA detector are used as a priori LLRs of the turbo decoder. For fair comparison with the benchmark schemes to be detailed in the following sections, a total of $P_{\text {TC-SCMA }}$ outer iterations are required for completing the iterative detection and decoding process. Furthermore, a total of $T=\sum_{p=1}^{P_{\mathrm{TC}-\mathrm{SCMA}}}\left(T_{\mathrm{MPA}, p}+T_{\mathrm{LBCJR}, p}\right)$ iterations are employed for performing either MPA detection or LBCJR turbo decoding, where $T_{\mathrm{MPA}, p}$ represents the number of iterations required for MPA detection in the $p$-th outer iteration, $1 \leq p \leq P_{\mathrm{TC}-\mathrm{SCMA}}$ and $T_{\mathrm{LBCJR}, p}$ represents the number of iterations required for LBCJR turbo decoding in the $p$-th outer iteration. For SDD, we have $P_{\mathrm{TC}-\mathrm{SCMA}}=1$. Hence, the subscript ' $p$ ' is omitted for $T_{\mathrm{LBCJR}, p}$ and $T_{\mathrm{MPA}, p}$ when describing SDD.

The input-output relationship of the MPA detector can be explicitly described by the factor graph [27] of Fig. 2, where the $f$-th symbol $x_{k}^{(f)}$ sent by user $k$, is represented by a 


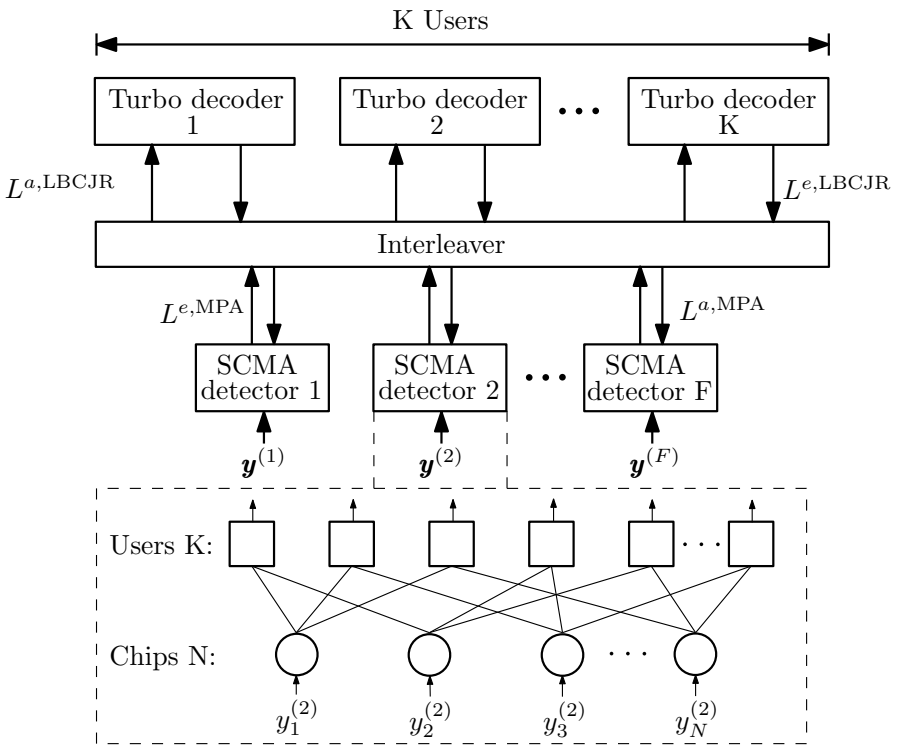

Fig. 2. Receiver schematic of the turbo-coded SCMA system.

user variable node (UVN) $j, 1 \leq j \leq K$, while the $N$ corresponding observations of the $f$-th symbol of all $K$ users in $\boldsymbol{y}^{(f)}$ are considered as $N$ chip check nodes (CCNs). In the following description, $(f)$ is omitted for simplicity, since the MPA detection of the $f$-th symbol and the MPA detection operations of all $F$ symbols are identical. As shown in Fig. 2, users 1,2 and 3 share the $i=1$-st CCN. Although these three users do interfere with each other during the 1-st $\mathrm{CCN}$, the information carried by the different CCNs conveyed by the three users can also be transferred from one $\mathrm{CCN}$ to another via the connections of the factor graph. Before we detail the operations of the MPA detector, we first define a pair of sets for characterizing the connections of the factor graph as

$$
\begin{aligned}
& \overline{\mathcal{K}}_{j}=\left\{i: 1 \leq i \leq N, e_{j i} \neq 0\right\}, j=1,2, \ldots, K \\
& \overline{\mathcal{C}}_{i}=\left\{j: 1 \leq j \leq K, e_{j i} \neq 0\right\}, i=1,2, \ldots, N,
\end{aligned}
$$

where we have $\left|\overline{\mathcal{K}}_{j}\right|=d_{x}$, representing the $d_{x}$ connections with UVN $j$, and $\left|\overline{\mathcal{C}}_{i}\right|=d_{c}$, giving the $d_{c}$ connections with $\mathrm{CCN} i$.

Additionally, the symbol set of user $k$ is expressed as $\mathcal{X}^{k}=$ $\left\{s_{1}^{k}, s_{2}^{k}, \ldots, s_{M}^{k}\right\}$, whereas the probability $\eta_{j, i}^{s_{m}^{j},(t)}$ is defined as the message sent from UVN $j$ to CCN $i$ during the $t$-th MPA iteration. More specifically, $\eta_{j, i}^{s_{m}^{j},(t)}$ is the probability that we have $x_{j}=s_{m}^{j}$, given all the messages received by the $j$-th UVN from all of its neighboring CCNs, excluding CCN $i$. By contrast, the message sent from CCN $i$ to UVN $j$ during the $t$-th MPA iteration, $\delta_{i, j}^{s_{m}^{j},(t)}$, is defined as the probability that $x_{j}=s_{m}^{j}$, given the specific messages received by CCN $i$ from all its neighboring UVNs, excluding $x_{j}$. Following [28] and with the aid of the factor graph of Fig. 2, the MPA detector can be described as follows.

First, for all $s_{m}^{k} \in \mathcal{X}^{k}$, and for any specific $(j, i)$ pairs, where $1 \leq j \leq K$ and $1 \leq i \leq N$, the probability $\eta_{j, i}^{s_{m}^{j}}$ is initialised as $\eta_{j, i}^{s_{m}^{j}},(0)=1 / M$. During the $t$-th $(t \geq 1)$ MPA iteration, the probability $\delta_{i, j}^{s_{m}^{j},(t)}$ can be expressed as

$$
\begin{aligned}
\delta_{i, j}^{s_{m}^{j},(t)}= & \sum_{\substack{\boldsymbol{x}_{[i]} \in \mathcal{X}_{[i] \backslash j}, x_{j}=s_{m}^{j}\\
}}\left(\prod_{x_{v} \in \boldsymbol{x}_{[i]} \backslash x_{j}} \eta_{j, i}^{x_{v},(t)}\right) \\
& \times p\left(y_{i} \mid \boldsymbol{x}_{[i]}, x_{j}=s_{m}^{j}\right), m=1,2, \ldots, M,
\end{aligned}
$$

where $x_{[i]}$ is a $d_{c}$-length vector containing the symbols sent by the $d_{c}$ users sharing the $i$-th subcarrier and $\mathcal{X}_{[i] \backslash j}$ represents the combination of symbols in the user codebooks for those specific $\left(d_{c}-1\right)$ users who share the $i$-th subcarrier, except for user $j$. Still referring to (7), $\prod_{x_{v} \in \boldsymbol{x}_{[i]} \backslash x_{j}} \eta_{j, i}^{x_{v},(t)}$ is the $a$ priori probability of a given $\boldsymbol{x}_{[i]}$ associated with $x_{j}=s_{m}^{j}$, while $p\left(y_{i} \mid \boldsymbol{x}_{[i]}\right)$ can be expressed as

$$
p\left(y_{i} \mid \boldsymbol{x}_{[i]}\right)=\frac{1}{2 \pi \sigma^{2}} \exp \left(-\frac{\left\|y_{i}-\sum_{j \in \overline{\mathcal{C}}_{i}} h_{j, i} x_{j, i}\right\|^{2}}{2 \sigma^{2}}\right),
$$

where we define $\overline{\mathcal{C}}_{i}$ as the set containing the indices of the specific users sending their information on the $i$-th subcarrier, as given in (6).

During the $(t+1)$-th MPA iteration, the values $\delta_{i, j}^{s_{m}^{j},(t)}$ are used for computing $\eta_{j, i}^{s_{m}^{j},(t+1)}$ using the expression of

$$
\eta_{j, i}^{s_{m}^{j},(t+1)}=\xi_{j, i} \prod_{v \in \overline{\mathcal{K}}_{j} \backslash i} \delta_{v, j}^{s_{m}^{j},(t)}, m=1,2, \ldots, M
$$

where $\xi_{j, i}$ is applied so that $\sum_{m=1}^{M} \eta_{j, i}^{s_{m}^{j},(t+1)}=1$.

Finally, when the maximum affordable number $T_{\mathrm{MPA}}$ of iterations has been exhausted, the likelihood of the $f$-th codeword symbol $x_{j}=s_{m}^{j}$ can be formulated as

$$
\eta_{j}^{s_{m}^{j}, f}=\prod_{v \in \overline{\mathcal{K}}_{j}} \delta_{v, j}^{s_{m}^{j},\left(T_{\mathrm{MPA}}\right), f}, j=1,2, \ldots, K .
$$


The extrinsic LLR of the $v$-th bit $\hat{b}_{v, j}$ in the codeword symbol $x_{j}$ can be expressed as

$$
L^{e, \mathrm{MPA}}\left(\hat{b}_{v, j}\right)=\ln \frac{\sum_{s_{m}^{j} \in \mathcal{X}^{j}, \hat{b}_{v, j}=0} \eta_{j}^{s_{m}^{j}, f}}{\sum_{s_{m}^{j} \in \mathcal{X}^{j}, \hat{b}_{v, j}=1} \eta_{j}^{s_{m}^{j}, f}}-L^{a, \mathrm{MPA}}\left(\hat{b}_{v, j}\right),
$$

where $L^{a, \mathrm{MPA}}\left(\hat{b}_{v, j}\right)$ is the a priori LLR of $\hat{b}_{v, j}$. When the bit LLRs of all $F$ symbols transmitted by user $k$ have been generated, de-interleaving is performed, and these deinterleaved extrinsic LLRs are input to the LBCJR turbo decoder [29], which can be expressed as

$$
L^{a, \operatorname{LBCJR}}\left(\hat{\boldsymbol{b}}_{k}\right)=\Pi^{-1}\left(L^{e, \operatorname{MPA}}\left(\hat{\boldsymbol{b}}_{k}\right)\right) .
$$

The LLRs $L^{a, \operatorname{LBCJR}}\left(\hat{\boldsymbol{b}}_{k}\right)$ are 'de-punctured' or 'de-repeated' according to the approaches adopted at the encoder to provide the upper decoder with both the systematic and parity $a$ priori LLRs $L^{a, \operatorname{LBCJR}}\left(\hat{\boldsymbol{b}}_{1, k}\right)$ and $L^{a, \operatorname{LBCJR}}\left(\hat{\boldsymbol{b}}_{2, k}\right)$, while the lower decoder is furnished with the parity a priori LLRs $L^{a, \operatorname{LBCJR}}\left(\hat{\boldsymbol{b}}_{3, k}\right)$ of user $k$, respectively.

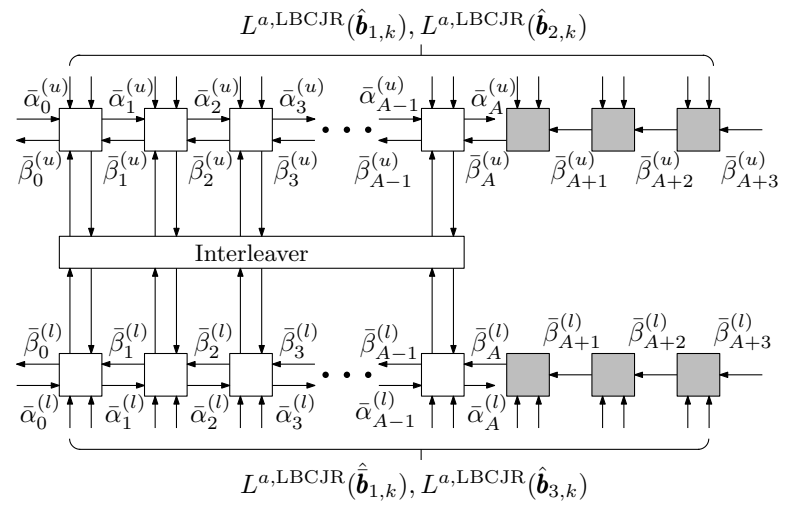

Fig. 3. The operations of the LBCJR turbo decoder.

The iterative LBCJR decoding process is illustrated in Fig. 3. To be more specific, in the $q$-th iteration, the upper and lower decoders operate alternately, where each decoder element of Fig. 3 generates the corresponding extrinsic LLRs $L^{e, \operatorname{LBCJR}}\left(\hat{\boldsymbol{b}}_{1, k}\right)$ or $L^{e, \operatorname{LBCJR}}\left(\hat{\overline{\boldsymbol{b}}}_{1, k}\right)$, which are interleaved or deinterleaved to provide the a priori LLRs $L^{a, \operatorname{LBCJR}}\left(\hat{\boldsymbol{b}}_{1, k}\right)$ or

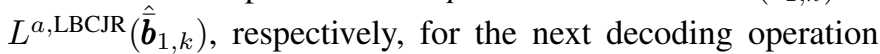
of the other decoder.

In addition to the LLRs described above, each decoder element of the $v$-th bit in Fig. 3 is provided with a vector of forward state metrics $\overline{\boldsymbol{\alpha}}_{v-1}^{(u)}$ or $\overline{\boldsymbol{\alpha}}_{v-1}^{(l)}$ as well as with a vector of backward state metrics $\overline{\boldsymbol{\beta}}_{v}^{(u)}$ or $\overline{\boldsymbol{\beta}}_{v}^{(l)}$, which are used for initiating the forward and backward processing recursions, respectively [23]. During the forward recursion, each successive decoder element in a left-to-right order is processed in each successive clock cycle. Simultaneously, the backward recursion activates the successive decoder element in a rightto-left order during successive clock cycles. Each successive decoder element passes a vector of state metric values $\overline{\boldsymbol{\alpha}}_{v}^{(u)}$, $\overline{\boldsymbol{\alpha}}_{v}^{(l)}, \overline{\boldsymbol{\beta}}_{v-1}^{(u)}$ or $\overline{\boldsymbol{\beta}}_{v-1}^{(l)}$ to the next decoder element in the forward or backward recursion, respectively.

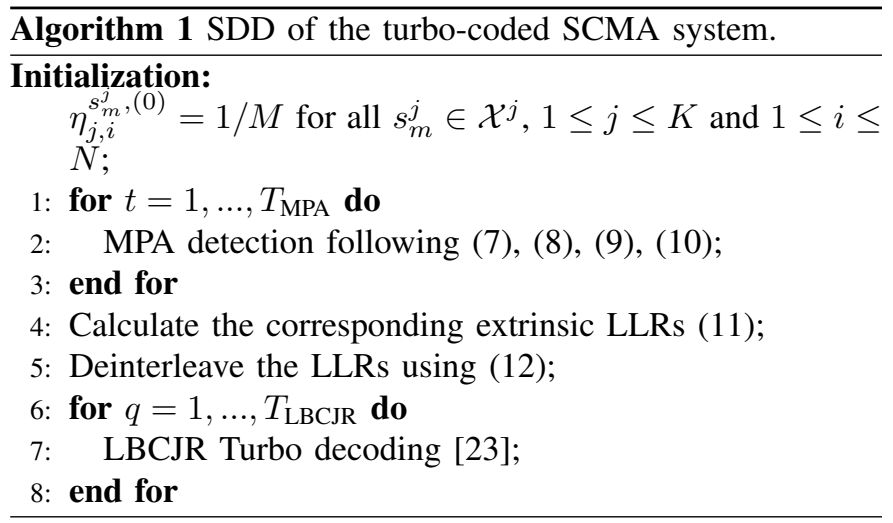

Furthermore, once the two recursions have crossed over, extrinsic LLRs are generated, as mentioned above, based on both the forward and backward state metrics. Here, we omit the calculation of the forward and backward state metrics for the sake of simplicity, but the details of these calculations can be found in [23]. After several iterations ${ }^{1}$, the transmitted bits $\hat{\boldsymbol{b}}_{k}$ of user $k$, are decoded according to the extrinsic LLRs. The SDD algorithm is summarised in Algorithm 1.

\section{B. Joint signal detection and decoding}

In contrast to SDD, the JDD scheme facilitates LLR exchange between the MPA detector and LBCJR turbo decoder in each outer iteration, which improves the BER performance, as will be demonstrated in Section IV. For the JDD, we employ $T_{\mathrm{MPA}, p}=T_{\mathrm{LBCJR}, p}=1$ for the $p$-th outer iteration.

Similar to SDD, after the same initialisation as that of the MPA detector of SDD, the JDD starts out by updating $\eta_{j, i}^{s_{m}^{j},(1)}$ based on (9) in the $p$-th outer iteration. The updated codeword probability $\eta_{j, i}^{s_{m}^{j},(1)}$ is converted to bit LLRs according to (11) and input to the upper turbo decoder of Fig. 3 as a priori LLRs. These LLRs are deinterleaved and forwarded to the lower decoder as the parity LLRs. After turbo decoding, the output extrinsic bit LLRs are entered into the MPA detector. Specifically, the $\bar{E}$ extrinsic bit LLRs are punctured or added to give $L^{e, \operatorname{LBCJR}}\left(\hat{\boldsymbol{b}}_{k}\right)$, which is input to the interleaver, giving $E$ a priori bit LLRs $L^{a, \mathrm{MPA}}\left(\hat{\boldsymbol{b}}_{k}\right)=\left[L^{a, \mathrm{MPA}}\left(\hat{b}_{1, j}^{1}\right), \cdots, L^{a, \mathrm{MPA}}\left(\hat{b}_{v, j}^{f}\right)\right.$, $\left.\cdots, L^{a, \operatorname{MPA}}\left(\hat{b}_{\log _{2} M, j}\right)\right]^{T}$ for the MPA detector, where $b_{v, j}$ is the $v$-th bit of symbol $s_{m}^{j}$. The $E$ LLRs are converted to $F=E / \log _{2} M$ a priori symbol probabilities for user $k$. For each SCMA symbol, the a priori symbol probability $\eta_{j}^{s_{m}^{j}}$ is calculated as

$$
\eta_{j}^{s_{m}^{j}}=\prod_{v=1}^{\log _{2} M} \frac{\exp \left(\left(1-b_{v, j}\right) L^{a, \mathrm{MPA}}\left(\hat{b}_{v, j}\right)\right)}{1+\exp \left(L^{a, \mathrm{MPA}}\left(\hat{b}_{v, j}\right)\right)} .
$$

The new $\eta_{j, i}^{s_{m}^{j}}$ of (13) is fed back to the MPA detector of Fig. 2 for MPA detection, as detailed in Section III-A.

The JDD algorithm is summarised in Algorithm 2.

${ }^{1} T_{\mathrm{LBCJR}}=8$ iterations may be required in order to achieve iterative decoding convergence to the best possible BER [29]. 


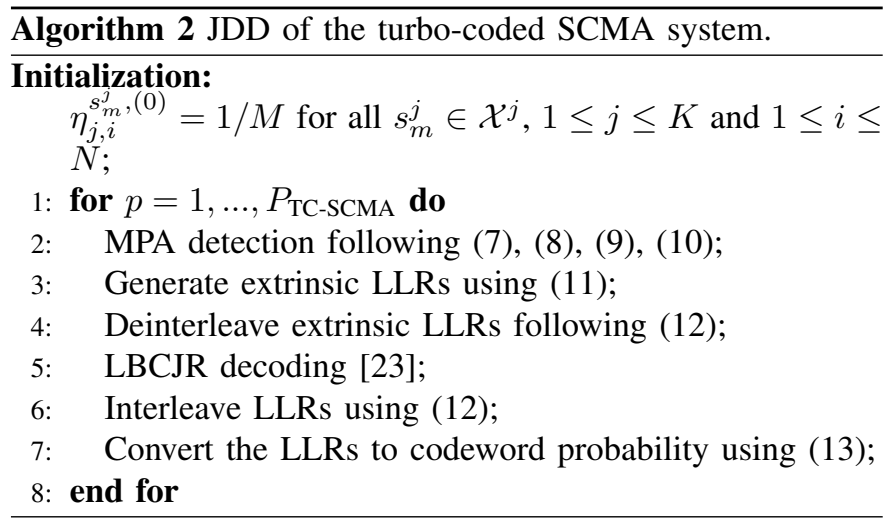

\section{SYSTEM PERFORMANCE AND HYBRID DETECTION AND DECODING (HDD)}

In this section, we analyse the performance of the turbocoded SCMA system employing either SDD or JDD in terms of their capacity upper bound, BER performance as well as convergence rate in Sections IV-A to IV-C. In the simulations, QPSK $(M=4)$ is used and $d_{x}=2$ is fixed for all simulation results. Hence, according to (2), we have $d_{c}=K d_{x} / N$. The code rate is $R \in[1 / 3,3 / 4]$. Furthermore, based on our EXIT chart analysis, we will advocate a pair of HDD schemes due to its BER vs. complexity advantage, as demonstrated in Section IV-D, based on its complexity comparison to that of SDD and JDD discussed in Section IV-E.

\section{A. Capacity of the SCMA system}

We first analyse the capacity of the SCMA UL, where $K$ users simultaneously transmit their $f$-th symbol to the BS in the $f$-th time slot. In the following derivations, we only employ the superscript $(f)$ when necessary. Since the signals are transmitted by $N$ orthogonal subcarriers of the SCMA system and received by $U$ receive antennas (RAs), the achievable rate is upper bounded by the discrete-input continuous-output memoryless channel (DCMC) capacity [30, 31]. Therefore, we may view the $N$ subcarriers as $N$ transmit antennas (TAs) of a conventional MIMO system and derive the DCMC capacity for the SCMA system following [31].

First, let us define the symbol set $\mathcal{X}_{[i]}$ as the combinational symbol set for the $d_{c}$ users who share the $i$-th subcarrier as

$$
\mathcal{X}_{[i]}=\left\{a_{1}, a_{2}, \cdots, a_{M^{d_{c}}}\right\} .
$$

Hence, we have $\boldsymbol{x}_{[i]}=a_{m} \in \mathcal{X}_{[i]}$. According to [31], the DCMC capacity (bits/subcarrier) can be expressed as

$$
\begin{aligned}
C= & \frac{1}{N d_{x}} \sum_{i=1}^{N} \max _{p\left(\boldsymbol{x}_{[i]}=a_{m}\right), a_{m} \in \mathcal{X}_{[i]}} \sum_{m=1}^{M^{d_{c}}} \int_{-\infty}^{+\infty} p\left(y_{i}, \boldsymbol{x}_{[i]}=a_{m}\right) \\
& \times \log _{2}\left(\frac{p\left(y_{i} \mid \boldsymbol{x}_{[i]}=a_{m}\right)}{\sum_{v=1}^{M^{d_{c}}} p\left(y_{i}, \boldsymbol{x}_{[i]}^{\prime}=a_{v}\right)}\right) \mathrm{d} y_{i}
\end{aligned}
$$

where $p\left(y_{i} \mid \boldsymbol{x}_{[i]}=a_{m}\right)$ is given by (8). Note that (15) is maximised in the case of equiprobable transmitted symbols,

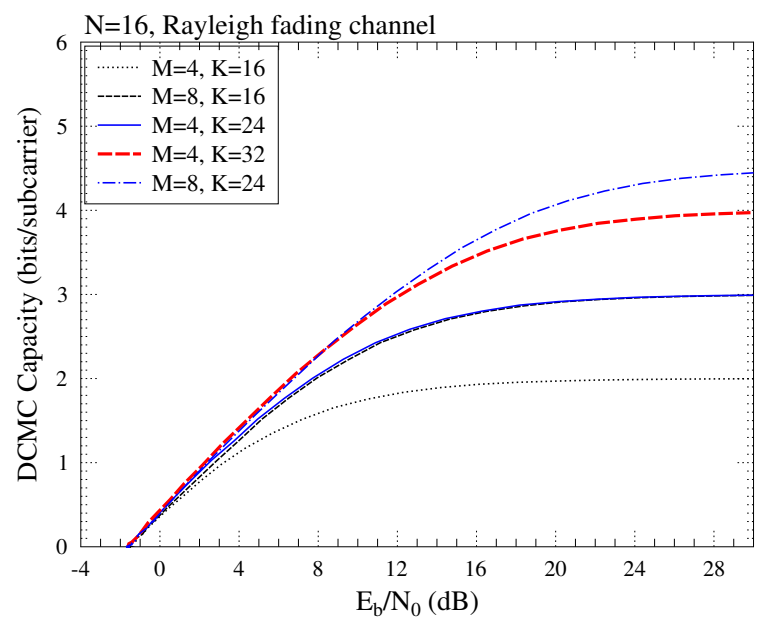

Fig. 4. Upper bound of the DCMC capacity of (18) for the $N=16$ turbocoded SCMA system, where $d_{x}=2$.

where we have $p\left(\boldsymbol{x}_{[i]}=a_{m}\right)=1 /\left(M^{d_{c}}\right), \forall m$. Hence, we arrive at

$$
\begin{aligned}
\log _{2} & \left(\frac{p\left(y_{i} \mid \boldsymbol{x}_{[i]}=a_{m}\right)}{\sum_{v=1}^{M^{d_{c}}} p\left(y_{i}, \boldsymbol{x}_{[i]}^{\prime}=a_{v}\right)}\right) \\
& =\log _{2}\left(\frac{p\left(y_{i} \mid \boldsymbol{x}_{[i]}=a_{m}\right)}{\sum_{v=1}^{M^{d_{c}}} p\left(y_{i} \mid \boldsymbol{x}_{[i]}^{\prime}=a_{v}\right) p\left(\boldsymbol{x}_{[i]}^{\prime}=a_{v}\right)}\right) \\
& =-\log _{2}\left(\frac{1}{M^{d_{c}}} \sum_{v=1}^{M^{d_{c}}} \frac{p\left(y_{i} \mid \boldsymbol{x}_{[i]}^{\prime}=a_{v}\right)}{p\left(y_{i} \mid \boldsymbol{x}_{[i]}=a_{m}\right)}\right) \\
& =\log _{2}\left(M^{d_{c}}\right)-\log _{2} \sum_{v=1}^{M^{d_{c}}} \exp \left(\Psi_{i}\right),
\end{aligned}
$$

where by substituting (4) and (8) into (16), $\Psi_{i}$ is expressed as

$$
\Psi_{i}=\frac{-\left|\left(\boldsymbol{x}_{[i]}-\boldsymbol{x}_{[i]}^{\prime}\right) \boldsymbol{h}_{[i]}+n_{i}\right|^{2}+\left|n_{i}\right|^{2}}{2 \sigma^{2}},
$$

with $h_{[i]}$ representing a $d_{c}$-length vector of the channel gains between the $i$-th subcarrier of the $d_{c}$ users sharing this subcarrier and the $\mathrm{BS}$, while $n_{i}$ is the $i$-th element in $\boldsymbol{n}$.

Hence, if the symbols are transmitted with equal probabilities, we arrive at an upper bound of the DCMC capacity expressed as

$$
\begin{aligned}
C_{\max }= & \lambda \log _{2} M- \\
& \frac{1}{d_{x} N M^{d_{c}}} \sum_{i=1}^{N} \sum_{m=1}^{M^{d_{c}}} \mathbb{E}_{\boldsymbol{h}, \boldsymbol{n}}\left[\log _{2} \sum_{v=1}^{M^{d_{c}}} \exp \left(\Psi_{i}\right)\right] .
\end{aligned}
$$

Using (18), Fig. 4 gives the normalised DCMC capacity $C_{\max }$ (bits/subcarrier) for an SCMA system obtained from (18). We can see that a higher normalised user load $\lambda$ results in a higher DCMC capacity bound, but at the cost of degraded BER performance, as will be demonstrated in Section IV-B. Another factor that influences the DCMC capacity bound is the SCMA modulation order $M$, as a higher order enables 


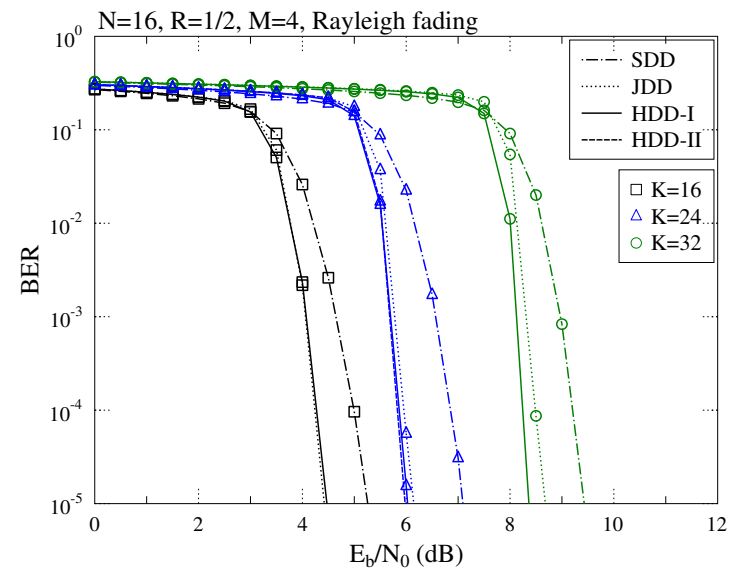

Fig. 5. BER performance of a turbo-coded SCMA system with $N=16$ for supporting different number of users over an uncorrelated Rayleigh fading channel, where the coding rate $R$ is fixed at $1 / 2$.

more non-zero information bits to be transmitted by a single SCMA codeword. Note that in Fig. 4, $M=4$ indicates QPSK is employed while $M=8$ represents 8QAM [4]. Furthermore, a turbo-coded system supporting $K=24$ users with $M=4$ achieves the same DCMC capacity bound compared to that supporting $K=16$ users with $M=8$, since the number of bits transmitted per subcarrier remains the same.

\section{B. Error correction performance of the turbo-coded SCMA system}

The BER performance of the turbo-coded SDD and JDDaided SCMA system having different $\lambda$ values for transmission over the AWGN channel is shown in Figs. 5 and 6, where we have $N=16, K=16,24$ or $32, F=512$ and $T=16$. We can see that compared to $\mathrm{SDD}$, up to $1 \mathrm{~dB}$ gain can be attained at a BER level of $10^{-5}$ by JDD as a benefit of exchanging extrinsic LLRs between the MPA detector and LBCJR decoder during each outer iteration. The highest gain is attained at a normalised user load of $\lambda=1$, where the multi-user interference is the least severe.

Naturally, the coding rate $R$ also influences the system performance, as seen by comparing Figs. 5 and 6 . Explicitly, the turbo-coded SCMA system using $R=1 / 3$ in Fig. 6 requires about $1 \mathrm{~dB}$ lower $E_{b} / N_{0}$ than that of $R=1 / 2$ shown in Fig. 5. To be more specific, $R=1 / 2$ is achieved by puncturing some of the information and parity bits [25]. While this redundancy reduction gives a higher useful data rate, it results in a slight performance degradation.

Fig. 7 compares the influence of the normalised user load $\lambda$ and of the coding rate $R$ on the turbo-coded system. Let us first define the normalised system load as $\omega=\lambda R=K R / N$, before investigating the relationship of $\lambda$ and $R$. As shown in Fig. 7, an $R=2 / 3$ turbo-coded system supporting $K=24$ users has the same normalised system load of $\omega=1$ as an $R=1 / 2$ turbo-coded system supporting $K=32$ users, but achieves better BER performance. However, the opposite is true for $\omega=3 / 4$ with $K=16, R=3 / 4$ and $K=24, R=1 / 2$

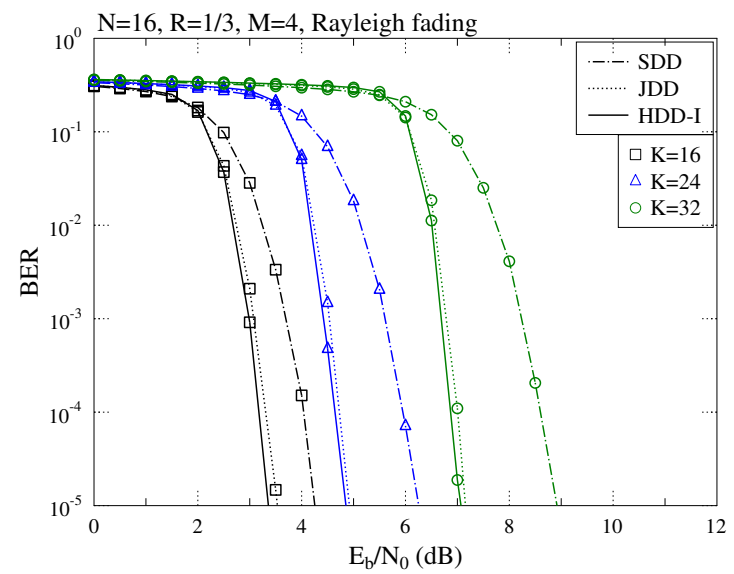

Fig. 6. BER performance of a turbo-coded SCMA system with $N=16$ for supporting different number of users over an uncorrelated Rayleigh fading channel, where the coding rate $R$ is fixed at $1 / 3$.

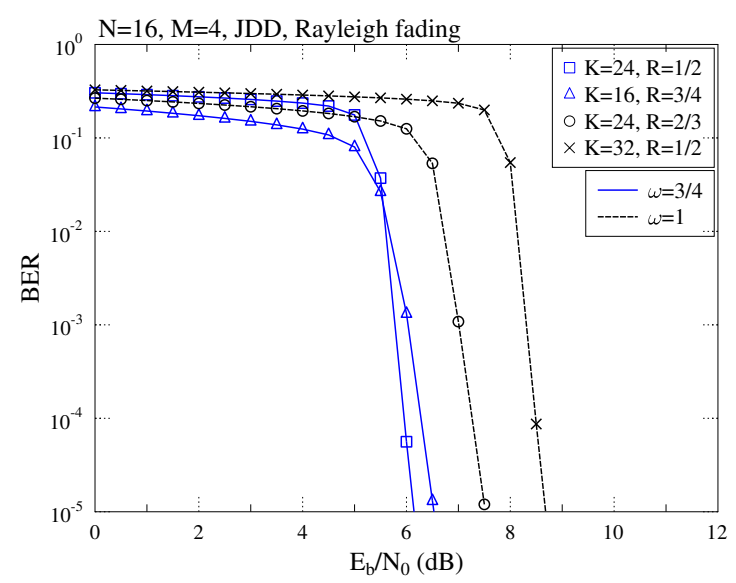

Fig. 7. BER performance of a turbo-coded SCMA system of different coding rates with $N=16$ for normalized user loads of $\lambda=1,1.5$ or 2 and system load $\omega=3 / 4$ or 1 over an uncorrelated Rayleigh fading channel.

turbo-coded SCMA system. Note that $R>1 / 3$ is achieved by puncturing some of the information and parity bits.

\section{EXIT chart analysis of the turbo-coded SCMA system}

Let us now employ EXIT charts for visualising the convergence behavior of our MPA and LBCJR schemes. Originally, EXIT charts were proposed for characterising the mutual information (MI) of a pair of concatenated components in a communication system. In this section, we employ EXIT charts for characterising the relationship between the a priori and extrinsic information of the MPA detector and the LBCJR decoder. First, following [32] and assuming that the a priori LLRs $L^{a, \text { MPA }}$ for the MPA detector obey a Gaussian random distribution with a mean of $\mu_{A}$ and a variance of $\sigma_{A}^{2}$ satisfying $\mu_{A}=\sigma_{A}^{2} / 2$, the conditional probability density function 
(PDF) associated with $L^{a, \mathrm{MPA}}\left(\hat{\boldsymbol{b}}_{k}\right)$ can be expressed as

$$
p_{a}(\zeta \mid X=x)=\frac{1}{\sqrt{2 \pi} \sigma_{A}} \exp \left(-\frac{\left(\zeta-\frac{\sigma_{A}^{2}}{2} x\right)^{2}}{2 \sigma_{A}^{2}}\right),
$$

where $x=1$ or -1 . Assuming $P(X=x)=1 / 2$, the MI between the a priori LLRs $L^{a, \mathrm{MPA}}\left(\hat{\boldsymbol{b}}_{k}\right)$ of the MPA detector and the turbo encoded bits $\hat{\boldsymbol{b}}_{k}$ can be expressed as

$$
\begin{aligned}
I_{a, \mathrm{MPA}}= & -\frac{1}{\sqrt{2 \pi} \sigma_{A}} \int_{-\infty}^{+\infty} \exp \left(-\frac{\left(\zeta-\frac{\sigma_{A}^{2}}{2}\right)^{2}}{2 \sigma_{A}^{2}}\right) \\
& \times \log _{2}\left[1+e^{-\zeta}\right] \mathrm{d} \zeta .
\end{aligned}
$$

By contrast, the MI between the extrinsic LLRs $L^{e, \mathrm{MPA}}\left(\hat{\boldsymbol{b}}_{k}\right)$ of the MPA detector and turbo encoded bits can be expressed as

$$
\begin{aligned}
I_{e, \mathrm{MPA}}= & \frac{1}{2} \cdot \sum_{u=-1,+1} \int_{-\infty}^{+\infty} p_{e}(\zeta \mid U=u) \\
& \times \log _{2} \frac{2 p_{e}(\zeta \mid U=u)}{p_{e}(\zeta \mid U=-1)+p_{e}(\zeta \mid U=1)} \mathrm{d} \zeta,
\end{aligned}
$$

where $p_{e}(\zeta \mid U=u)$ is obtained by using the classic MonteCarlo method. From (21), we can express $I_{e, \mathrm{MPA}}$ as a function of $I_{a, \mathrm{MPA}}$ and $E_{b} / N_{0}$ defined as

$$
I_{e, \mathrm{MPA}}=\mathcal{T}_{\mathrm{MPA}}\left(I_{a, \mathrm{MPA}}, E_{b} / N_{0}\right) .
$$

In contrast to $I_{e, \mathrm{MPA}}$, the extrinsic information transfer function of the turbo decoder is independent of $E_{b} / N_{0}$, since the only input of the decoder is the soft-interleaved extrinsic LLR sequence $L^{e, \mathrm{MPA}}\left(\hat{\boldsymbol{b}}_{k}\right)$ of the MPA detector, as shown in Fig. 2. Therefore, the extrinsic information transfer characteristic is defined as

$$
I_{e, \mathrm{LBCJR}}=\mathcal{T}_{\mathrm{LBCJR}}\left(I_{a, \mathrm{LBCJR}}\right),
$$

where $I_{a, \mathrm{LBCJR}}$ is the MI between the turbo-coded bits $\boldsymbol{b}_{k}$ and a priori LLRs $L^{a, \mathrm{LBCJR}}\left(\hat{\boldsymbol{b}}_{k}\right)$ of the LBCJR decoder.

Fig. 8 shows the EXIT chart of an $N=16$ turbo-coded SCMA system supporting $K=24$ users for transmission over the uncorrelated Rayleigh fading channel. This will be used for determining the most beneficial activation order of both the SCMA MPA detector and of the LTE turbo decoder, so that we can achieve a receiver processing latency reduction at a given BER performance, as detailed in Section IV-D.

\section{Improved scheduling of detection and decoding: $H D D$}

According to the EXIT chart of Fig. 8, we propose a pair of HDD schemes, which improves the activation order of the SCMA MPA detector and LBCJR turbo decoder, for reducing the complexity and latency of detection and decoding without degrading BER performance. To be more specific, HDD aims for a relatively high initial MI at $I_{e, \mathrm{LBCJR}}=0$ and a wider open tunnel, which would lead to expedited convergence overall. Therefore, HDD commences with several MPA iterations first for attaining a high initial MI. The extrinsic LLRs at its output are entered into the LBCJR decoder as a priori LLRs.

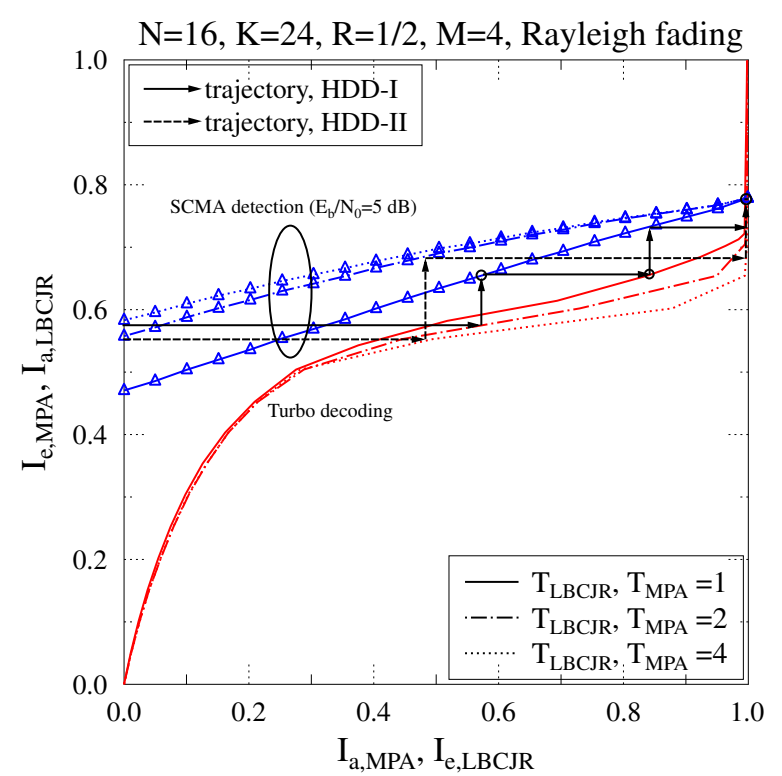

Fig. 8. The EXIT chart for the LBCJR turbo decoder and for an SCMA MPA detector using $N=16$ subcarriers for supporting $K=24$ users for transmission over an uncorrelated Rayleigh fading channel. The triangles indicate the SCMA MPA curves, while the lines without symbols are the turbo decoder curves. The curves are not shown for more than $T_{\mathrm{MPA}}=4$, as $T_{\mathrm{MPA}}=8$ becomes indistinguishable from $T_{\mathrm{MPA}}=4$.

Furthermore, we can see from Fig. 8 that the MI obtained from $T_{\mathrm{MPA}}=1,2$ and 4 SCMA MPA iterations eventually converges to $I_{e, \mathrm{MPA}}=0.77$ at $I_{e, \mathrm{LBCJR}}=1$. Therefore, the marginal improvement attained by further MI exchanges remains rather limited, despite increasing the detection complexity of the receiver. Therefore, the JDD philosophy may be adopted after the first several iterations. To be more specific, we discuss a pair of design examples ${ }^{2}$ that detail our proposed lowcomplexity HDD using the EXIT chart of Fig. 8.

We refer to our first design example as HDD-I. In the case of a turbo-coded SCMA system using $N=16, F=512$, we first employ $T_{\mathrm{MPA}, 1}=4$ iterations for SCMA MPA detection, which gives a high MI at $I_{e, \mathrm{LBCJR}}=0$, as shown in Table II. Note that even though eight MPA iterations gives a slightly better MI compared to four iterations, this improvement remains rather marginal, despite its doubled complexity. The following $T_{\mathrm{LBCJR}, 1}=2$ iterations are employed for turbo decoding, relying on iterative LLR exchange. Following this, four iterations are employed for JDD activation, as shown in Table II. The HDD-I scheduling is also visualized by the staircase shaped decoding trajectory seen in Fig. 8. In this way, only $T=10$ iterations are required for completing iterative detection and decoding, hence achieving early termination of the iterations.

An alternative design, namely HDD-II employs a lower number of iterations for MPA detection but a higher number of turbo decoding iterations. Specifically, we first employ $T_{\mathrm{MPA}, 1}=2$ iterations for MPA detection, followed by

\footnotetext{
${ }^{2}$ The inspirational suggestion of HDD-II by an anonymous reviewer to include for comparison is gratefully acknowledged by the authors.
} 
TABLE II

SCHEDULING OF DIFFERENT ITERATIVE DETECTION AND DECODING SCHEMES FOR THE TURBO-CODED SCMA SYSTEM.

\begin{tabular}{|c|c|c|c|c|c|c|c|c|c|c|c|c|c|c|c|c|c|}
\hline & Iterations $(T)$ & 1 & 2 & 3 & 4 & 5 & 6 & 7 & 8 & 9 & 10 & 11 & 12 & 13 & 14 & 15 & 16 \\
\hline \multirow{2}{*}{ SDD } & SCMA & $\checkmark$ & $\checkmark$ & $\checkmark$ & $\checkmark$ & $\checkmark$ & $\checkmark$ & $\checkmark$ & $\checkmark$ & - & - & - & - & - & - & - & - \\
\hline & Turbo & - & - & - & - & - & - & - & - & $\checkmark$ & $\checkmark$ & $\checkmark$ & $\checkmark$ & $\checkmark$ & $\checkmark$ & $\checkmark$ & $\checkmark$ \\
\hline \multirow{2}{*}{ JDD } & SCMA & $\checkmark$ & - & $\checkmark$ & - & $\checkmark$ & - & $\checkmark$ & - & $\checkmark$ & - & $\checkmark$ & - & $\checkmark$ & - & $\checkmark$ & - \\
\hline & Turbo & - & $\checkmark$ & - & $\checkmark$ & - & $\checkmark$ & - & $\checkmark$ & - & $\checkmark$ & - & $\checkmark$ & - & $\checkmark$ & - & $\checkmark$ \\
\hline \multirow{2}{*}{ HDD-I } & SCMA & $\checkmark$ & $\checkmark$ & $\checkmark$ & $\checkmark$ & - & - & $\checkmark$ & - & $\checkmark$ & - & - & - & - & - & - & - \\
\hline & Turbo & - & - & - & - & $\checkmark$ & $\checkmark$ & - & $\checkmark$ & - & $\checkmark$ & - & - & - & - & - & - \\
\hline \multirow{2}{*}{ HDD-II } & SCMA & $\checkmark$ & $\checkmark$ & - & - & - & - & $\checkmark$ & $\checkmark$ & - & - & - & - & $\checkmark$ & - & - & - \\
\hline & Turbo & - & - & $\checkmark$ & $\checkmark$ & $\checkmark$ & $\checkmark$ & - & - & $\checkmark$ & $\checkmark$ & $\checkmark$ & $\checkmark$ & - & $\checkmark$ & - & - \\
\hline
\end{tabular}

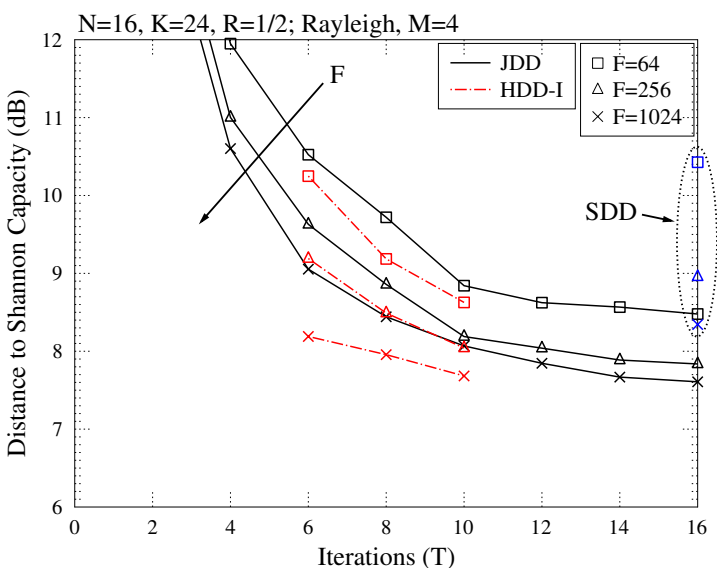

Fig. 9. The $E_{b} / N_{0}$ distance to Shannon capacity of SDD, JDD and HDD-I for an $N=16, K=24$ turbo-coded SCMA system in each iteration at a BER level of $10^{-3}$ over the uncorrelated Rayleigh fading channel, where $R=1 / 2$ and $M=4$.

$T_{\mathrm{LBCJR}, 1}=4$ iterations for LBCJR turbo decoding. The same scheduling is repeated for the following six iterations, i.e. $T_{\mathrm{MPA}, 2}=2$ and $T_{\mathrm{LBCJR}, 2}=4$. Finally, we employ JDD for the final two iterations, as shown in Table II. In total, $T=14$ iterations are required for completing the detection and decoding operations. The BER performance of Fig. 5 shows very similar performances for both HDD schemes, but compared to HDD-I, HDD-II requires lower detection complexity, as will be shown in Section IV-E. Note that LBCJR turbo decoding can be performed serially for $E$ coded bits $^{3}$, hence a higher decoding latency is imposed. By contrast, MPA detection can be implemented in parallel for $F$ symbols. Therefore, different HDD schemes can be selected based on the specific practical design targets. For the rest of the simulation results, to avoid overlapping curves, we use the HDD-I scheme only as a design example.

Furthermore, the continuous-input continuous-output memoryless channel (CCMC) Shannon capacity [33], which approaches $E_{b} / N_{0}=-1.59 \mathrm{~dB}$ at zero capacity, is considered as a benchmark of a coded system. Hence, Fig. 9 compares the $E_{b} / N_{0}$ distance to the CCMC Shannon capacity of the three different activation schemes at each iteration and shows the influence of frame length $F$ at a BER level of $10^{-3}$. Fig. 10 shows the influence of the normalized user-load $\lambda$. We can see that HDD-I is capable of achieving almost the same $E_{b} / N_{0}$ as

${ }^{3}$ For LTE turbo codes, multiple LBCJR turbo decoders may operate in parallel to reduce latency, depending on the frame length $E$ [17].

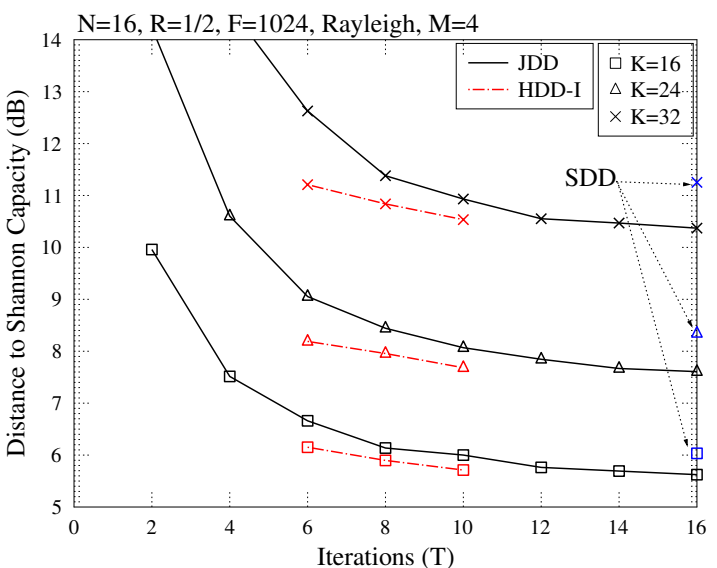

Fig. 10. The $E_{b} / N_{0}$ distance to Shannon capacity of SDD, JDD and HDD-I for the turbo-coded SCMA system supporting different number of users in each iteration at a BER level of $10^{-3}$ over the uncorrelated Rayleigh fading channel, where $N=16, R=1 / 2$ and $M=4$.

JDD. Also, the $E_{b} / N_{0}$ values of HDD-I at ten iterations are all slightly above the JDD points at sixteen iterations, despite its reduced number of iterations. This reduces the complexity as well as power consumption at the receiver and improves detection latency. The $E_{b} / N_{0}$ performance of HDD-I in Figs. 9 and 10 provide us with design guidelines for the practical implementation of the receiver, where low-latency detection is attained at a given SNR based on our EXIT chart.

\section{E. Complexity Analysis}

The receiver complexity is quantified by the number of additions, multiplications, and $\max ^{*}$ [17] calculations. The complexity of the LBCJR turbo decoder decoding $A$ bits for the LTE turbo code is defined in [29] as

$$
\mathcal{C}_{\mathrm{LBCJR}}=171 A K T_{\mathrm{LBCJR}}
$$

while the complexity of the MPA detector can be expressed as [34]

$$
\begin{aligned}
\mathcal{C}_{\mathrm{MPA}}= & {\left[\left(2 d_{c}+4\right) M^{d_{c}} d_{x}+\left(d_{x}-2+M^{d_{c}-1}\right) M d_{x}-d_{x}\right] } \\
& \times F K T_{\mathrm{MPA}}+M\left(d_{x}-1\right) K F .
\end{aligned}
$$

Therefore, the total complexity of the different decoding and detection schemes is the sum of $\mathcal{C}_{\mathrm{LBCJR}}$ in (24) and $\mathcal{C}_{\mathrm{MPA}}$ in (25). Table III compares the complexity of SDD, JDD, HDDI and HDD-II for turbo-coded SCMA systems for different $\lambda$ values. We can see that by employing the HDD-I scheme, 
a complexity reduction of up to $25 \%$ is achieved in the case of $\lambda=2$, since only $T_{\mathrm{LBCJR}}=4$ and $T_{\mathrm{MPA}}=6$ iterations are required for LBCJR decoding and MPA detection, respectively. By contrast, when the normalised user load is high, for example, $\lambda=2$, the complexity of MPA detection becomes dominant. In this case, HDD-II achieves the lowest complexity among the four schemes in Table III, which is only $64 \%$ of JDD/SDD.

\section{AdAPtive Turbo-COded SCMA System}

In this section, we propose an adaptive turbo-coded SCMA system design for transmission over correlated fading channels by harmonizing the user load $\lambda$, modulation order $M$ and coding rate $R$ based on different instantaneous SNR (iSNR). In this way, an increased BPS throughput can be achieved at a given BER. The system design of the adaptive turbo-coded SCMA system is detailed in Section V-A, followed by the corresponding adaptive system performance in Section V-B.

\section{A. Adaptive system design}

In an adaptive modulation system, $D$ transmission modes achieving different BPS throughputs are available at the transmitter, where the receiver estimates the iSNR and then determines the most appropriate transmission mode to be employed by the transmitter. According to [35], given the channel gains of the $f$-th transmitted symbol as $\bar{h}_{f}$, the iSNR $\gamma_{i}$ associated with transmitting a frame comprising $F$ symbols can be expressed as

$$
\gamma_{i}=\frac{\|\overline{\boldsymbol{h}}\|^{2}}{F} \gamma=\frac{\|\overline{\boldsymbol{h}}\|^{2}}{2 F \sigma^{2}},
$$

where $\overline{\boldsymbol{h}}=\left[\overline{\boldsymbol{h}}_{1}^{T}, \overline{\boldsymbol{h}}_{2}^{T}, \cdots, \overline{\boldsymbol{h}}_{F}^{T}\right]^{T}$.

The iSNR $\gamma_{i}$ is compared to various SNR thresholds at the receiver, which are required by the $D$ fixed transmission modes to attain the target BER level. The mode selection requirements can be expressed as

$$
\text { Mode Selection }=\left\{\begin{array}{ccc}
\text { Mode 1, } & \text { if } & \gamma_{i} \leq \rho_{1} \\
\text { Mode 2, } & \text { if } & \rho_{1}<\gamma_{i}<\rho_{2} \\
\vdots & & \vdots \\
\text { Mode D, } & \text { if } & \gamma_{i} \geq \rho_{D-1} .
\end{array}\right.
$$

In this way, when the channel conditions are more favorable, a higher throughput may be achieved by selecting a higherBPS mode, whereas a more robust but lower-BPS transmission mode will be employed in poorer channels.

In this section, we select $D=3$ transmission mode candidates, as listed in Table IV. Our adaptive turbo-coded SCMA system, jointly assigns different normalised user loads, coding rates and modulation orders. As demonstrated in Section IV-B, the turbo-coded SCMA system is capable of supporting normalized user loads up to $\lambda=2$. Therefore, two loads are selected, i.e., $\lambda=1.5$ or 2 . The coding rates selected for the proposed adaptive system are $R=1 / 4,1 / 3$ and $1 / 2$, whereas the modulation order of the different modes is either $M=2$ or $M=4$.

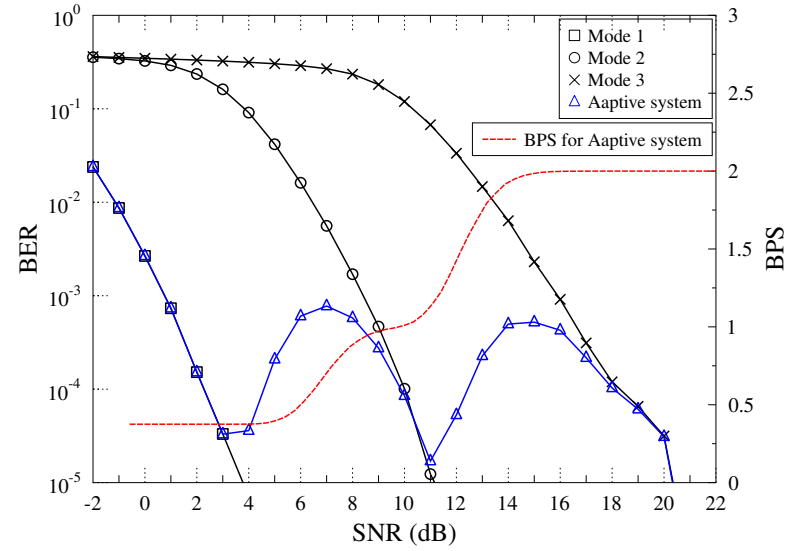

Fig. 11. BER performance of the turbo-coded SCMA system over the ETU channel with a Doppler frequency of $70 \mathrm{~Hz}$ employing fixed or adaptive modulation design, where $N=16$ is fixed for all scenarios and the parameters of Table IV are used.

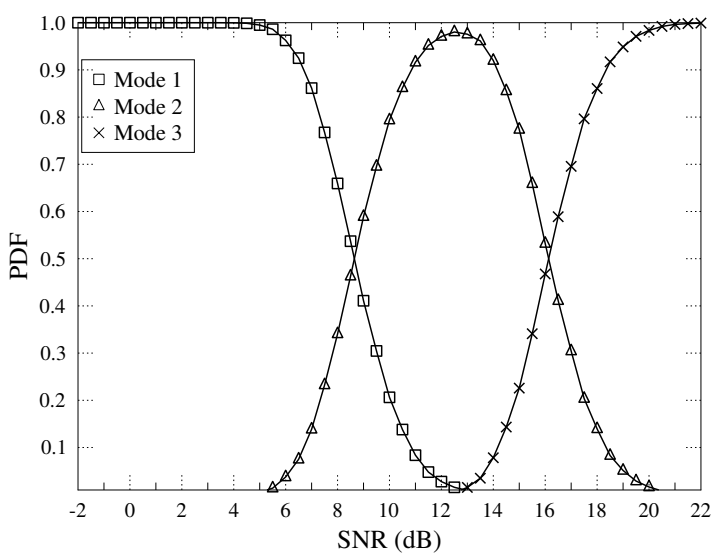

Fig. 12. The PDF of the different modes in Table IV in the adaptive turbocoded SCMA system.

\section{B. Performance of the adaptive turbo-coded SCMA system}

In this section, the performance of the proposed adaptive turbo-coded SCMA system employing HDD-I is evaluated for transmission over the extended typical urban (ETU) channel model with a Doppler frequency of $f=70 \mathrm{~Hz}$ [36]. In this case, the mobile velocity $v$ can be calculated as [37]

$$
v=\frac{f c}{f_{c}}=8.75 \mathrm{~m} / \mathrm{s},
$$

where $f_{c}=2.4 \mathrm{GHz}$ is the carrier frequency employed in the IEEE 802.11 standard [38] and $c=3 \times 10^{8} \mathrm{~m} / \mathrm{s}$ is the speed of light.

Fig. 11 shows the BER performance of the three different modes of Table IV and of the adaptive design switching amongst the three modes. Here, the switching thresholds of the different modes are determined by the average SNR required for achieving a BER of $10^{-3}$, as shown in Fig. 11. For the three candidate modes conceived in Table IV, we have a pair of mode-switching thresholds, namely $\rho_{1}=8.4 \mathrm{~dB}$ and $\rho_{2}=15.9 \mathrm{~dB}$. We can see that by adopting our adaptive 
TABLE III

COMPLEXITY OF THE DIFFERENT DETECTION AND DECODING SCHEMES, WHERE $N=16, M=4, d_{x}=2$.

\begin{tabular}{l|c|c|c|c|c|c|c}
\hline \multirow{2}{*}{ Scheme } & \multirow{2}{*}{ Iterations } & \multicolumn{2}{|c}{ Complexity $\left(\times 10^{7}\right)$ when $F=512$} & \multicolumn{3}{c}{ Complexity $\left(\times 10^{7}\right)$} & when $F=1024$ \\
\cline { 3 - 8 } & & $K=16$ & $K=24$ & $K=32$ & $K=16$ & $K=24$ & $K=32$ \\
\hline \hline SDD / JDD & 16 & 3.00 & 15.51 & 89.46 & 6.00 & 31.01 & 178.93 \\
\hline HDD-I & 10 & 1.97 & 11.22 & 66.54 & 3.94 & 22.42 & 133.08 \\
\hline HDD-II & 14 & 2.44 & 10.53 & 57.04 & 4.87 & 21.07 & 114.08 \\
\hline
\end{tabular}

TABLE IV

TRANSMISSION MODES FOR THE ADAPTIVE TURBO-CODED SCMA SYSTEM, WHERE $N=16, d_{x}=2$ AND $d_{c}=K d_{x} / N$.

\begin{tabular}{|c|c|c|c|c|}
\hline Mode & $\begin{array}{c}\text { Normalised user load } \\
\lambda\end{array}$ & $\begin{array}{c}\text { Code rate } \\
R \\
\end{array}$ & $\begin{array}{l}\text { Modulation order } \\
\qquad M\end{array}$ & $\begin{array}{c}\text { BPS } \\
\lambda R \log _{2} M \\
\end{array}$ \\
\hline 1 & 1.5 & $\frac{1}{4}$ & 2 & $\frac{3}{8}$ \\
\hline 2 & 1.5 & $\frac{4}{1}$ & 4 & 1 \\
\hline 3 & 2 & $\frac{3}{2}$ & 4 & 2 \\
\hline
\end{tabular}

transmission scheme, the BPS throughput varies from $3 / 8$ to 2 , depending on the choice of transmission modes. The corresponding PDF of the three modes in the adaptive system at different SNRs is shown in Fig. 12, which shows the dominant transmission mode at a specific SNR. It is also notable that at the threshold SNRs of $8.4 \mathrm{~dB}$ and $15.9 \mathrm{~dB}$, the adjacent modes share an equal probability of 0.5 .

\section{CONCLUSIONS}

An EXIT-chart-aided HDD design was conceived for turbocoded SCMA systems, which reduces the complexity of conventional JDD by up to $36 \%$, without degrading BER performance. Furthermore, we proposed an adaptive turbo-coded SCMA system, where the transmitter selects the transmission mode according to the near-instantaneous channel conditions. The adaptive turbo-coded SCMA system harmonizes the user load, coding rate as well as modulation order so that a higher data rate is achieved in more favorable channel conditions by employing high-BPS transmission modes, whereas the BER target performance is still maintained in the face of poorer channels by employing more robust low-BPS transmission modes. Finally, our adaptive system design principle can be readily extended to SCMA systems in combination with other channel coding schemes such as LDPC codes and polar codes.

\section{REFERENCES}

[1] L. Dai, B. Wang, Z. Ding, Z. Wang, S. Chen, and L. Hanzo, "A survey of non-orthogonal multiple access for 5G," IEEE Communications Surveys Tutorials, vol. 20, no. 3, pp. 2294-2323, 2018.

[2] Y. Liu, Z. Qin, M. Elkashlan, Z. Ding, A. Nallanathan, and L. Hanzo, "Nonorthogonal multiple access for $5 \mathrm{G}$ and beyond," Proceedings of the IEEE, vol. 105, no. 12, pp. 2347-2381, 2017.

[3] C. Bockelmann, N. Pratas, H. Nikopour, K. Au, T. Svensson, C. Stefanovic, P. Popovski, and A. Dekorsy, "Massive machine-type communications in 5G: Physical and MAC-layer solutions," IEEE Communications Magazine, vol. 54, no. 9, pp. 59-65, 2016.

[4] Y. Liu, L.-L. Yang, and L. Hanzo, "Spatial modulation aided sparse code-division multiple access," IEEE Transactions on Wireless Communications, vol. 17, no. 3, pp. 1474-1487, 2018.

[5] Y. Liu, L.-L. Yang, P. Xiao, H. Haas, and L. Hanzo, "Spatial modulated multicarrier sparse code-division multiple access," IEEE Transactions on Wireless Communications, vol. 19, no. 1, pp. 610-623, 2020.

[6] J. Wang, Z. Zhang, and L. Hanzo, "Joint active user detection and channel estimation in massive access systems exploiting Reed-Muller sequences," IEEE Journal of Selected Topics in Signal Processing, vol. 13, no. 3, pp. 739-752, 2019.
[7] H. Mu, Z. Ma, M. Alhaji, P. Fan, and D. Chen, "A fixed low complexity message pass algorithm detector for up-link SCMA system," IEEE Wireless Communications Letters, vol. 4, no. 6, pp. 585-588, 2015.

[8] J. Dai, K. Niu, C. Dong, and J. Lin, "Improved message passing algorithms for sparse code multiple access," IEEE Transactions on Vehicular Technology, vol. 66, no. 11, pp. 9986-9999, 2017.

[9] Y. Wu, S. Zhang, and Y. Chen, "Iterative multiuser receiver in sparse code multiple access systems," in 2015 IEEE International Conference on Communications (ICC), pp. 2918-2923, IEEE, 2015.

[10] B. Xiao, K. Xiao, S. Zhang, Z. Chen, B. Xia, and H. Liu, "Iterative detection and decoding for SCMA systems with LDPC codes," in 2015 International Conference on Wireless Communications \& Signal Processing (WCSP), pp. 1-5, IEEE, 2015.

[11] K. Han, Z. Zhang, J. Hu, and J. Chen, "A high performance joint detection and decoding scheme for LDPC coded SCMA system," in 2016 IEEE Globecom Workshops (GC Wkshps), pp. 1-6, IEEE, 2016.

[12] J. Dai, K. Niu, Z. Si, C. Dong, and J. Lin, "Polar-coded non-orthogonal multiple access," IEEE Transactions on Signal Processing, vol. 66, no. 5, pp. 1374-1389, 2017.

[13] Z. Pan, E. Li, L. Zhang, J. Lei, and C. Tang, "Design and optimization of joint iterative detection and decoding receiver for uplink polar coded SCMA system," IEEE Access, vol. 6, pp. 52014-52026, 2018.

[14] L. Yuan, J. Pan, N. Yang, Z. Ding, and J. Yuan, "Successive interference cancellation for LDPC coded nonorthogonal multiple access systems," IEEE Transactions on Vehicular Technology, vol. 67, no. 6, pp. 54605464, 2018

[15] J. Dai, K. Niu, and J. Lin, "Iterative gaussian-approximated message passing receiver for MIMO-SCMA system," IEEE Journal of Selected Topics in Signal Processing, vol. 13, no. 3, pp. 753-765, 2019.

[16] Z. B. K. Egilmez, L. Xiang, R. G. Maunder, and L. Hanzo, "The development, operation and performance of the $5 \mathrm{G}$ polar codes," IEEE Communications Surveys \& Tutorials, vol. 22, no. 1, pp. 96-122, 2019.

[17] L. Xiang, M. F. Brejza, R. G. Maunder, B. M. Al-Hashimi, and L. Hanzo, "Arbitrarily parallel turbo decoding for ultra-reliable low latency communication in 3GPP LTE," IEEE Journal on Selected Areas in Communications, vol. 37, no. 4, pp. 826-838, 2019.

[18] B. Tahir, S. Schwarz, and M. Rupp, "BER comparison between convolutional, turbo, LDPC, and polar codes," in 2017 24th International Conference on Telecommunications (ICT), pp. 1-7, IEEE, 2017.

[19] M. A. Lema, A. Laya, T. Mahmoodi, M. Cuevas, J. Sachs, J. Markendahl, and M. Dohler, "Business case and technology analysis for $5 \mathrm{G}$ low latency applications," IEEE Access, vol. 5, pp. 5917-5935, 2017.

[20] J. Sachs, L. A. Andersson, J. Araújo, C. Curescu, J. Lundsjö, G. Rune, E. Steinbach, and G. Wikström, "Adaptive 5G low-latency communication for tactile Internet services," Proceedings of the IEEE, vol. 107, no. 2, pp. 325-349, 2018.

[21] L. Hanzo, L.-L. Yang, E. Kuan, and K. Yen, Single-and multi-carrier DS-CDMA: multi-user detection, space-time spreading, synchronisation, standards and networking. John Wiley \& Sons, 2003.

[22] T. Keller and L. Hanzo, "Adaptive multicarrier modulation: A convenient framework for time-frequency processing in wireless communications," Proceedings of the IEEE, vol. 88, no. 5, pp. 609-640, 2000.

[23] L. Hanzo, T. Liew, B. Yeap, R. Tee, and S. X. Ng, Turbo coding, turbo equalisation and space-time coding: EXIT-chart-aided near-capacity designs for wireless channels, vol. 22. John Wiley \& Sons, 2011. 
[24] O. Y. Takeshita, "Permutation polynomial interleavers: An algebraicgeometric perspective," IEEE Transactions on Information Theory, vol. 53, no. 6, pp. 2116-2132, 2007.

[25] 3GPP TS 36.212 version 8.8.0 Release 8, "LTE evolved universal terrestrial radio access (E-UTRA) multiplexing and channel coding," Jan 2010.

[26] L. Li, Z. Ma, P. Z. Fan, and L. Hanzo, "High-dimensional codebook design for the SCMA down link," IEEE Transactions on Vehicular Technology, vol. 67, no. 10, pp. 10118-10122, 2018.

[27] R. Hoshyar, F. P. Wathan, and R. Tafazolli, "Novel low-density signature for synchronous CDMA systems over AWGN channel," IEEE Transactions on Signal Processing, vol. 56, no. 4, pp. 1616-1626, 2008.

[28] M. C. Davey and D. MacKay, "Low-density parity check codes over GF( q)," IEEE Communications Letters, vol. 2, pp. 165-167, June 1998

[29] R. G. Maunder, "A fully-parallel turbo decoding algorithm," IEEE Transactions on Communications, vol. 63, no. 8, pp. 2762-2775, 2015.

[30] J. H. Van Lint, R. M. Wilson, and R. M. Wilson, A course in combinatorics. Cambridge university press, 2001.

[31] S. X. Ng and L. Hanzo, "On the MIMO channel capacity of multidimensional signal sets," IEEE Transactions on Vehicular Technology, vol. 55, no. 2, pp. 528-536, 2006

[32] M. El-Hajjar and L. Hanzo, "EXIT charts for system design and analysis," IEEE Communications Surveys \& Tutorials, vol. 16, no. 1, pp. 127-153, 2013.

[33] W. C. Lee, "Estimate of channel capacity in Rayleigh fading environment," IEEE Transactions on Vehicular Technology, vol. 39, no. 3, pp. 187-189, 1990.

[34] L. Yang, Y. Liu, and Y. Siu, "Low complexity message passing algorithm for SCMA system," IEEE Communications Letters, vol. 20, no. 12, pp. 2466-2469, 2016.

[35] A. Svensson, "An introduction to adaptive QAM modulation schemes for known and predicted channels," Proceedings of the IEEE, vol. 95 no. 12, pp. 2322-2336, 2007.

[36] E. U. T. R. Access, "User equipment (UE) radio transmission and reception," 3GPP TS, vol. 36, p. V10, 2011.

[37] R. Steele and L. Hanzo, Mobile radio communications: Second and third generation cellular and WATM systems: 2nd. IEEE Press-John Wiley, 1999.

[38] Q. Ni, "Performance analysis and enhancements for IEEE $802.11 \mathrm{e}$ wireless networks," IEEE Network, vol. 19, no. 4, pp. 21-27, 2005. 